\title{
Investigation of sensory thresholds in Cavalier King Charles Spaniels with and without Chiari-like malformations and syringomyelia
}

\author{
Courtney R. Sparks ${ }^{1}$ | Angela Gorney ${ }^{1}$ | Kim Williams ${ }^{1}$ | Emily H. Griffith ${ }^{2}$ | \\ Sofia Cerda-Gonzalez ${ }^{3}$ | B. Duncan X. Lascelles ${ }^{1,4,5}$ ๑ Natasha J. Olby ${ }^{1,5}$ ()
}

${ }^{1}$ Department of Clinical Sciences, College of Veterinary Medicine, North Carolina State

University, Raleigh, North Carolina

${ }^{2}$ Department of Statistics, North Carolina State University, Raleigh, North Carolina

${ }^{3}$ Department of Clinical Sciences, College of Veterinary Medicine, Cornell University, Ithaca, New York

${ }^{4}$ Comparative Medicine Institute, North Carolina State University, Raleigh, North Carolina

${ }^{5}$ Comparative Pain Research and Education Center, North Carolina State University, Raleigh, North Carolina

\section{Correspondence}

Natasha J. Olby, 1060 William Moore Drive, Raleigh, NC 27606.

Email: njolby@ncsu.edu

Funding information

American Cavalier King Charles Spaniel Club Charitable Trust, Grant/Award Number: 02162-MOU; American Kennel Club Canine Health Foundation
Background: Cavalier King Charles Spaniels (CKCS) suffer pain associated with Chiari-like malformation and syringomyelia (CMSM). People suffer from a similar condition and describe numerous sensory abnormalities. Sensory changes have not been quantified in affected CKCS.

Objectives: To use quantitative sensory testing (QST) to quantify thermal and mechanical thresholds in CKCS and to compare QST in dogs with and without syringomyelia (SM).

Animals: Forty-four CKCS.

Methods: Prospective study. Dogs underwent neurological examinations and craniocervical magnetic resonance imaging (MRI). Thermal testing was performed over the humerus and thorax $(n=32)$; mechanical testing was performed on the paw and neck $(n=44)$. Latencies, thresholds, and response rates were compared with presence and severity of SM on MRI, presence of pain reported by the owner and pain identified on examination.

Results: Thirty dogs had SM, 30 were painful on examination, 29 were owner-reported symptomatic. Thermal and mechanical variables were not significantly different based on presence or severity of SM. Dogs with pain on examination had decreased mechanical thresholds on the paw $(0.38 \mathrm{~kg}, \mathrm{SD}=0.18)$ and neck $(2.05 \mathrm{~kg}, \mathrm{SD}=0.74)$ compared to thresholds of dogs without pain on examination on the paw $(0.60 \mathrm{~kg}, \mathrm{SD}=0.30)$ and neck $(2.72 \mathrm{~kg}, \mathrm{SD}=0.57 ; P=.021)$.

Conclusions and Clinical Importance: Mechanical and thermal sensitivity does not appear to be related to the presence of SM, but mechanical sensitivity appears to be related to the presence of pain and clinical signs. Mechanical testing may be useful for assessing sensory abnormalities during clinical trials.

\section{KEYWORDS}

neuropathic pain, paresthesia, phantom scratch, syrinx

\section{1 | INTRODUCTION}

Pain syndromes in dogs are challenging to identify and manage because of the difficulty of inferring behaviors in dogs. Veterinarians

Abbreviations: CKCS, Cavalier King Charles Spaniels; CM, Chiari-like malformation; CM1, Chiari-type 1 malformation; CSF, cerebrospinal fluid; MRI, magnetic resonance imaging; NCSU, North Carolina University; QST, quantitative sensory testing; SM, syringomyelia must rely on owner observations and physical examination findings to assess the efficacy of treatments, and frequently a mismatch is present in these observations. Specifically, Chiari-like malformation and syringomyelia (CMSM) is a disease complex that causes a neuropathic pain syndrome in Cavalier King Charles Spaniels (CKCS). This condition arises from a congenital malformation that results in a relatively small caudal fossa with respect to the brain causing crowding of the cerebellum and brainstem. ${ }^{1-7}$ Many CKCS with CM also develop 
syrinxes within the spinal cord (syringomyelia, SM) because of disruption of cerebrospinal fluid (CSF) flow. Commonly, owners of affected CKCS report signs of phantom scratching, crying out in pain, rubbing of the face and ears, pain on defecation, reluctance to play, collar sensitivity, and aversion to being touched on the head, ${ }^{8}$ and these signs are thought to result from SM disrupting sensory pathways. ${ }^{9-11}$

Chiari-type 1 malformation (CM1) in humans is similar to CMSM in CKCS and involves descent of the cerebellum and brainstem through the foramen magnum. Chiari-type 1 malformation in humans also is commonly associated with the development of SM, with an estimated $65 \%-80 \%$ of patients developing both conditions. ${ }^{12,13}$ People with CM1 describe a wide range of signs including numbness, neck pain, headaches, memory loss, aphasia, and even depression. ${ }^{14}$ Pain and sensory deficits in human CM1 patients have been quantified using thermal and mechanical sensory testing, and patient drawn pain maps. ${ }^{14-17}$ Thermohypoesthesia and decreased sensory perception are common findings in people with $\mathrm{CM} 1$ and $\mathrm{SM},{ }^{1,15}$ but the presence of pain in human patients clearly indicates that sensory changes can involve both gain as well as loss of function. Although the majority of outcome assessments in people with neuropathic pain rely on the patient's description of sensations based on questionnaires or phone call follow-ups, the use of quantitative sensory testing (QST; thermal and mechanical) has been validated using test-retest and interobserver reliability. ${ }^{18}$ Furthermore, 1 study indicated that the duration of sensory deficits quantified by QST before surgery was the best predictor of surgical outcome in patients with SM. ${ }^{15}$

Treatment options for dogs with CMSM focus on pain management, controlling CSF production, and surgical decompression of the caudal fossa. Frequently, these options fail to completely alleviate signs of scratching and pain in these dogs. A major challenge in determining whether treatment options are successful is the difficulty in documenting and quantifying neuropathic pain in these dogs. Questionnaire tools completed by owners have been developed, ${ }^{8,19-21}$ but more objective quantification using QST is lacking. Thermal and mechanical testing has been shown to be reliable and discriminatory in dogs with other pain disorders. ${ }^{22-29}$ Our main objective was to use thermal and mechanical stimuli to quantify sensory thresholds in a cohort of CKCS and to compare sensory thresholds in dogs with and without SM. We hypothesized that CKCS affected with SM would exhibit decreased thermal latencies and mechanical thresholds as compared to dogs without SM.

\section{2 | MATERIALS AND METHODS}

\section{1 | Dogs and pain phenotyping}

Client-owned CKCS were prospectively recruited, both clinically affected and unaffected dogs, to North Carolina State University (NCSU) Veterinary Hospital. Dogs were required to be $\geq 15$ months of age, purebred, and healthy enough to undergo anesthesia for MRI. Health status was based on laboratory findings (CBC and serum biochemistry profile) performed within 2 weeks of anesthesia and a physical examination performed on the day of anesthesia; skin was examined carefully for evidence of primary skin disease as an explanation for scratching. A neurological examination also was performed, and the presence and location of pain elicited by spinal palpation was recorded as well as any scratching. Pain on neurological examination was defined as the presence of avoidance behavior or vocalization upon palpation of the neck and back. Any patient with a comorbidity (owner-reported, discovered upon physical examination, or previously noted in patient records) that potentially could cause pain unrelated to CMSM (eg, osteoarthritis) was excluded from the study. Owners completed previously developed questionnaires to determine affected status. ${ }^{8}$ All procedures were approved by NCSU Animal Use and Care Committee (IACUC protocol number 15-003-O) and owners signed informed consent forms.

\section{2 | Quantitative sensory testing}

All testing was performed in the same location. Dogs were introduced to the testing room and habituated to the handlers for 10 minutes before starting testing. Behavioral responses were defined before testing and animals were lightly restrained by a handler during testing in a way that did not hinder the dog's ability to react to the applied stimulus. Each dog was allowed to remain in a comfortable position (sitting or lying down) to minimize movement and avoid misidentification of behavioral responses. The same investigator (Courtney Sparks) applied the stimuli, recorded latencies and thresholds for all dogs, and was blinded to questionnaire information and MRI results that were performed before testing.

Owners were asked to withhold 2 consecutive doses of pain medications before testing, but owner refusal based on severity of clinical signs was noted and did not result in exclusion. Latencies to heat and cold were tested using a handheld thermal probe (NTE-2A, Physitemp instruments) with a $13 \mathrm{~mm}$ diameter surface (Figure 1) set at $46^{\circ} \mathrm{C}$ and $6^{\circ} \mathrm{C}$. Hair was clipped, creating a skin testing area approximately $15 \mathrm{~mm}$ in diameter at each site before testing and baseline skin temperature was recorded. A rest period (5-7 minutes) was allotted after hair clipping and the probe (with cover) was placed on the testing sites to allow for acclimation before applying temperature. Thermal sensory testing was performed laterally on the fore limb at the mid humeral level (called humerus) and lateral thorax bilaterally for each dog (Figure 1). For each timed trial, the probe was applied directly to the skin and held in place until the dog exhibited a response or a maximum of 30 or 60 seconds was reached for heat and cold, respectively. Behaviors that reflected conscious perception of the stimulus were deemed responses and included vocalization, head turning toward the probe, and escape behavior. If the maximum time was reached without a response, the trial was recorded as no response. Each trial was performed in triplicate separated by 1 minute intervals at every location. The pattern of testing was consistent for all dogs beginning with heat applied sequentially to the right humerus, right thorax, left humerus, and left thorax and followed by cold application in the same fashion. Latency was recorded as the duration, in seconds, it took the dog to respond during the timed trial and reported for each site by calculating the average of the triplicate testing and the response rate for each site (the percentage of trials in which the dog responded within the time limit) also was recorded. 
(A)

(B)

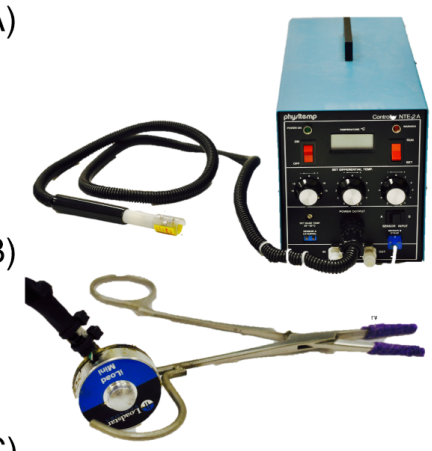

(C)

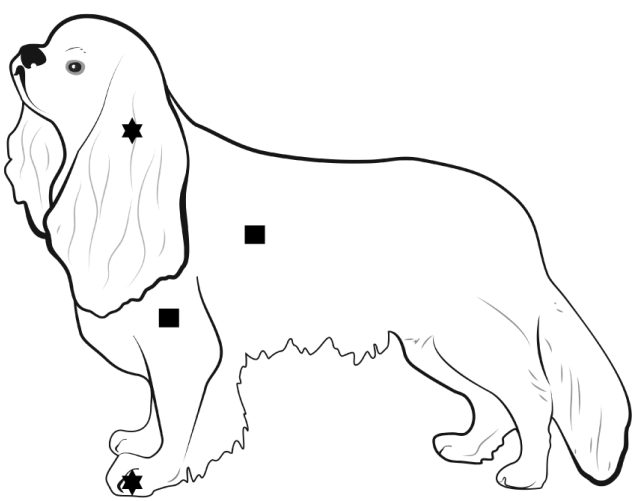

FIGURE 1 Quantitative sensory testing instruments and locations; (A) handheld Physitemp thermal probe used for heat and cold testing; (B) hemostratic forceps with a digital load cell used for mechanical testing. Thumb pressure was applied over the central button of the load cell; (C) testing locations for thermal (black squares) and mechanical testing (black stars)

Custom hemostatic forceps equipped with a digital load cell were used for quantification of mechanical thresholds ${ }^{30}$ (Figure 1). Testing was performed on the lateral digit of the front paws (the forceps were placed over the toe applying pressure to all tissues) and on the neck (skin was tented and grasped with the forceps), bilaterally. For the neck testing location, the skin was gently grasped, tented, and held steadily until the dogs remained still before introducing the mechanical stimulus. As pressure was applied to the load cell of the forceps, measurements were taken at a rate of $66 \mathrm{~Hz}$ and recorded with LoadVue software (Windows, ver. 3.0, Loadstar Sensors, Mountain View, California). Pressure was applied at a consistent rate of increase until a response was elicited or until a maximum force was reached (4 kg). The threshold was defined as the amount of force $(\mathrm{kg})$ required to elicit a response (eg, vocalization, escape behavior). The pattern of testing was the same for each dog with the following sequence: right paw, right neck, left paw, and left neck. All dogs were assigned feasibility scores based on behavior and ease of testing, and scores ranged from 0 (no difficulties) to 5 (impossible).

\section{3 | Magnetic resonance imaging protocol}

All dogs underwent MRI of the brain and cervical spinal cord using a 1.5 Tesla unit (Siemens Medical Solutions USA, Inc, Malvern, Pennsylvania). Anesthesia was induced using fentanyl ( $4 \mu \mathrm{g} / \mathrm{kg}$ IV, West-Ward Pharmaceuticals, Cherry Hill, New Jersey) as a premedication followed by propofol (4-6 mg/kg IV, Zoetis, Parsippany, New Jersey). Dogs were maintained under anesthesia using an inhaled isoflurane (Piramal
Enterprise Limited, Mumbai, India) and oxygen mixture. Dogs were positioned in ventral recumbency with their necks extended during the MRI. Acquired sequences included T2-weighted and proton density (PD) sagittal images, T2-weighted and PD transverse images of the cervical and cranial thoracic spine and brain.

\subsection{Magnetic resonance imaging assessment}

Images in a Digital Imaging and Communications format were analyzed using Horos Medical Imaging Software (Open Source software, https://www.horosproject.org/). Analysis was performed at least 3 months after the MRI was obtained, and sensory threshold data were not reviewed at the time of image analysis. All assessments were performed by 1 investigator (Courtney Sparks) and periodic checks on consistency were performed for $10 \%$ of cases by another author (Sofia Cerda-Gonzalez). A categorical assessment was done to determine the presence or absence of syrinx (defined as linear T2 hyperintensity on sagittal images $>2 \mathrm{~mm}$ in diameter). The maximum syrinx diameter was measured on transverse images. The presence of CM was determined by evidence of cerebellar indentation, impaction, or herniation through the foramen magnum on sagittal images.

\subsection{Statistical analysis}

All analyses were performed using JMP software (JMP Pro 12.2.0, SAS Version 9.4, Cary, North Carolina). Summary statistics were generated on the presence of SM, maximum SM diameter, presence of owner-reported signs, bilateral thermal latencies and mechanical thresholds, and response rates at each site tested. A Shapiro-Wilk test was used to assess normality of continuous data. Mean and SD calculations were reported for normally distributed data. Otherwise, median and range were reported. The relationship between sensory thresholds (latencies and response rates) and the presence (categorized as yes or no) and severity of SM (quantified by maximum SM diameter) was examined. In addition, latencies and response rates were compared with owner-reported signs, (symptomatic versus asymptomatic), and the presence of pain on neurological examination (yes or no). Wilcoxon rank sum tests were used when latencies and thresholds were compared with categorical data. Linear regression was used to model the relationship between continuous variables. All binary categorical data were compared using contingency tables and chi-square tests for association with Fisher's exact tests used when there were $<5$ observations in a category. The Benjamini-Hochberg procedure was used to correct for multiple comparisons. A $P$ value of $<.05$ was considered statistically significant.

\section{3 | RESULTS}

Fifty-six dogs were enrolled. Owner participation was voluntary, and therefore, the population was not considered random. The age range of dogs included in the study was 15 months to 11 years. There were 12 females, 16 spayed females, 8 males, and 20 neutered males. One patient had an adverse skin reaction during thermal testing (hyperemia) and was removed from the study. Another patient was removed 
from analysis because of the discovery of a brain tumor on MRI. Nine of 56 dogs (16\%) were imaged before involvement in the study and digital copies were provided by the owners. Magnetic resonance imaging studies performed at other hospitals were assessed for image quality and adequate sequences before enrollment. Two of 9 dogs imaged before enrollment were removed from analysis because their diagnosis status (no SM) was determined $>6$ months before sensory testing and therefore could not be confirmed at the time of testing. Another 3 dogs were removed because of difficulty in performing QST (high feasibility scores) or the presence of osteoarthritis. The remaining 4 had been imaged a median of 26 months (range, 6-32 months) before QST. A feasibility score $\geq 3$ (low feasibility) when performing QST resulted in exclusion from the study ( $\mathrm{n}=5$ dogs; score $3=3$ dogs, score $4=2$ dogs, and score $5=0$ ). One dog was excluded because of an orthopedic comorbidity and another dog was removed because the MRI was performed before caudal fossa decompression surgery and therefore diagnosis status of SM could not be confirmed at the time of sensory testing. Failure of owner compliance to return for sensory testing after MRI resulted in removal of 1 patient, leaving the total number of dogs analyzed at 44 . The thermal probe failed toward the end of the study and therefore 32 dogs were tested using temperature whereas all 44 underwent mechanical testing. Fourteen dogs were on medications at the time of enrollment and 7 owners complied by withholding 2 doses of medications before testing but 2 owners administered medications the night before but not the morning of, and 5 owners gave medications the morning of testing. Medications included gabapentin ( $n=9)$, omeprazole $(n=9)$, tramadol $(n=4)$, nonsteroidal anti-inflammatory drugs $(n=3)$, and pregabalin $(n=1)$. Seven dogs were receiving a combination of medications at the time of enrollment. The remaining 30 dogs were untreated at the time of testing.

\section{1 | Pain phenotype}

Owners reported 29/44 dogs as symptomatic, all 29/44 with scratching and 24/44 also showing signs of pain. Pain was present on neurological examination in 30 dogs, 22 of which were reported as symptomatic by the owners.

\section{2 | Magnetic resonance imaging findings}

All dogs had CM; 30/44 dogs had SM. The maximum syrinx diameter ranged from 0 to $9.5 \mathrm{~mm}$ (median, $4.2 \mathrm{~mm}$ ). Syringomyelia was present in 22 of the 29 owner-reported symptomatic dogs and in 8 owner-reported asymptomatic dogs and in 22 of 30 dogs with pain on neurological examination and in 8 of 14 with no pain on neurological examination. Cohort characteristics of dogs with and without SM are provided in Table 1.

\subsection{Sensory thresholds}

Thermal latencies were measured in 32 dogs and 44 dogs underwent mechanical testing. Feasibility scores are reported in Table 2. All modalities were assessed for normality. The neck mechanical thresholds and heat thermal latencies were normally distributed. The latencies for heat for the entire cohort of dogs averaged 17.0 seconds (SD, 7.5 seconds) at the humerus and 17.3 seconds (SD, 7.5 seconds) at the thorax. For cold, the latencies ranged from 0.49 to 52.3 seconds (median, 19.3 seconds) and 0.64 to 60 seconds (median, 29.0 seconds) for the humerus and thorax, respectively. The median response rate out of 3 trials for heat was $71 \%$ (range, $8.33 \%-100 \%$ ) and for cold was 83.33\% (range, 8.33\%-100\%). Response rates for each thermal testing location are shown in Supporting Information Table 1. The thresholds for mechanical testing averaged $2.26 \mathrm{~kg}(\mathrm{SD}, 0.75 \mathrm{~kg}$ ) for the neck and ranged from 0.13 to $1.44 \mathrm{~kg}$ (median, $0.4 \mathrm{~kg}$ ) for the lateral digit of the paw. All dogs responded to mechanical testing on the paw and $94 \%$ (83/88, total of left and right sides) responded on the neck.

No relationship was found between thermal or mechanical variables and the presence or severity of SM (Figure 2, $P>.05$, data for severity not shown). Dogs differentiated by pain on neurological examination had similar thermal variables as did dogs without pain, but they had significantly decreased thresholds to mechanical testing on the paw ( $p_{\text {adj }}=0.021$ ) and neck ( $p_{\text {adj }}=0.021$; Figure 3$)$. Thermal latencies and response rates were not different in dogs that were owner-reported symptomatic or not (Figure 4, $P>$.05). However, symptomatic dogs had decreased mechanical thresholds on the paw $\left(p_{\text {raw }}=0.022 ; p_{\text {adj }}=0.13\right)$ and neck $\left(p_{\text {raw }}=0.042 ; p_{\text {adj }}=0.13\right)$ compared to asymptomatic dogs, but the relationship disappeared after adjusting for multiple comparisons (Figure 4).

\section{4 | DISCUSSION}

In our study, we performed QST using mechanical ( $n=44)$ and thermal ( $n=32$, hot and cold) stimuli in a cohort of CKCS. Currently, no information is available on quantified sensory dysfunction comparing CKCS with and without CMSM. Our results showed no significant difference in latencies, thresholds or response rates, thermal or mechanical, in dogs with and without SM or in dogs that were owner-reported symptomatic versus asymptomatic but did show a correlation of mechanical thresholds to neurological examination findings.

Quantitative sensory testing has been used extensively in human CM1 patients and the results display a complicated mix of paresthesias, anesthesia, and allodynia. In 1 study, CM1 patients described an increase in number and area of painful sites on pain drawings but also experienced thermohypoesthesia of the face and body. ${ }^{17}$ Another study found that both SM and CM1 patients had anesthesia to heat

TABLE 1 Cohort characteristics of dogs with and without SM

\begin{tabular}{|c|c|c|c|}
\hline & No SM $(n=14)$ & $\mathrm{SM}(\mathrm{n}=30)$ & $P$ value \\
\hline Age (years) (median, range) & $4,1-8$ & $4,1-11$ & .499 \\
\hline $\operatorname{Sex}(M, M N, F, F S)$ & $3,6,4,1$ & $4,9,6,11$ & .226 \\
\hline Owner-reported symptomatic (n, \%) & $7,50 \%$ & $22,73 \%$ & .177 \\
\hline Pain on neurologic examination (n, \%) & $8,57 \%$ & $22,73 \%$ & .316 \\
\hline
\end{tabular}


TABLE 2 Number of dogs assigned to each feasibility score for dogs with SM and no SM

\begin{tabular}{|c|c|c|c|}
\hline & Feasibility sco & & \\
\hline & $\begin{array}{l}0 \\
\text { (No difficulty) }\end{array}$ & $\begin{array}{l}1 \\
\text { (Mild difficulty) }\end{array}$ & $\begin{array}{l}2 \\
\text { (Moderate difficulty) }\end{array}$ \\
\hline Syringomyelia $(n=30)$ & 14 & 12 & 4 \\
\hline No syringomyelia ( $n=14$ ) & 5 & 6 & 3 \\
\hline
\end{tabular}

and cold as well as vibration deficits that were of greater magnitude in the CM1 patients. ${ }^{15}$ An important distinction regarding the use of QST in people and dogs is the interpretation of the response. In humans, detection thresholds for the stimulus and the pain threshold for the same stimulus can be differentiated clearly by the subject. This is much more difficult to do in dogs. The behaviors we used to determine threshold were more likely to represent a response to a noxious stimulus but could simply mean the stimulus was perceived. All dogs were tested in the same way and we have not attempted to differentiate between perception and noxious thresholds.

The behaviors reported by owners of dogs with CMSM suggest that the dogs could be more sensitive to stimuli. However, the possibility that they have hypoesthesia (decreased sensitivity) is much harder to determine from their behavior. Furthermore, it may be the case that some dogs show decreased sensitivity whereas others show increased sensitivity or perhaps within a dog there may be bidirectional sensory changes (thermohypoesthesia and mechanical hyperalgesia) as seen in human medical literature. These complexities could explain the lack of associations found in our study. Indeed, the difficulty in interpreting behavioral responses both makes it challenging to understand the pathophysiology of the disease and to assess the efficacy of therapies for this disease. Currently, the use of owner assessments, collecting a detailed history, and physical and neurological examinations are used to monitor CKCS with CMSM. We attempted
(A)

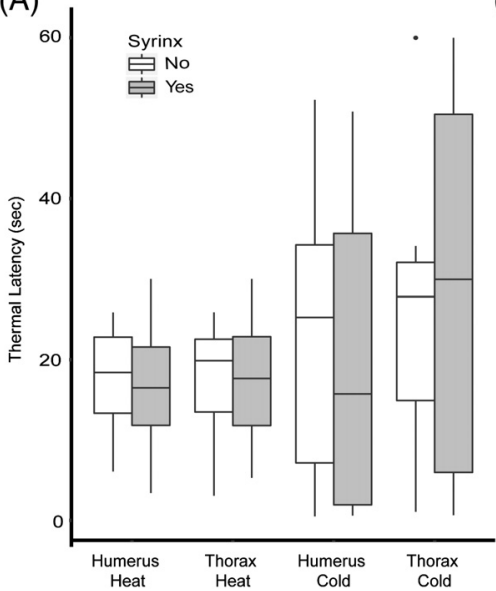

(B) 4

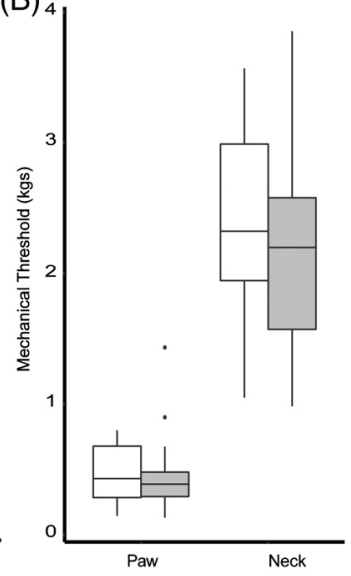

FIGURE 2 Box-plots showing thermal latencies (A) and mechanical thresholds (B) in dogs with and without syringomyelia. For thermal testing, every location is displayed as an average of left and right sides and triplicate trials per dog. For mechanical testing, left and right sides were averaged and every dog was tested once per location. The bottom and top of the boxes are the first and third quartiles, the line inside the box is the second quartile (median), the ends of the whiskers represent the lowest data within $1.5 \times$ IQR of the lower quartile and the highest data within $1.5 \times$ IQR of upper quartile. Dots represent outliers to collect QST data as a continuous measure of sensory dysfunction to complement our current, broader scales for pain.

Quantitative sensory testing has been used in dogs including studies in research colonies of beagles ${ }^{27,28}$ as well as client-owned healthy dogs $^{31}$ and in those with osteoarthritis ${ }^{25,29}$ and spinal cord injury. ${ }^{24,32}$ Various QST modalities (thermal and mechanical) have been employed in previous work. Mechanical stimuli include touch, pressure, and vibration, and the information is transmitted through mechanoreceptors in the skin and travels along myelinated axons through the dorsal columnmedial lemniscal pathway. Commonly, von Frey filaments have been used in humans with $\mathrm{SM}^{15,16}$ and in various studies of pain in dogs. ${ }^{23,25-29,32,33}$ The use of von Frey mechanical testing has been found to be feasible and reliable ${ }^{31,33}$ and in 1 study identified different thresholds in normal dogs compared to dogs with acute spinal cord injury. ${ }^{32}$ However, a lack of association between normal dogs and dogs with chronic pain also has been noted using von Frey techniques. ${ }^{23}$ Additionally, vibration testing is used in humans with $\mathrm{SM}^{15}$ and neuropathic pain, as another form of mechanical stimulation. ${ }^{34}$ No data have been reported on vibration testing in dogs and this approach may be considered for future work. In our study, mechanical testing was performed using specialized calibrated hemostatic forceps used in our laboratory previously. ${ }^{24}$ These forceps were developed to evaluate skin sensation after the administration of peripheral nerve blocks. ${ }^{30}$ This method was chosen based on ease of use at novel testing sites in the neck. A drawback of this technique, as for any mechanical testing technique, is variability in rate of application of pressure delivered by the
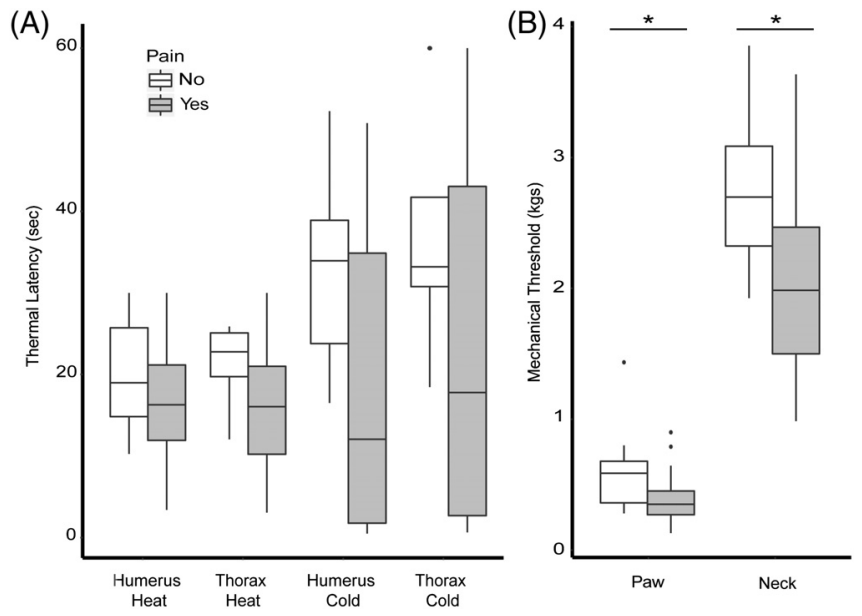

FIGURE 3 Box-plot showing thermal latencies (A) and mechanical thresholds (B) in dogs differentiated by pain on neurological examination. For thermal testing, every location is displayed as an average of left and right sides and triplicate trials per dog. For mechanical testing, left and right sides were averaged and every dog was tested once per location. Refer to Figure 2 legend for a description of the box-plot construct. * Indicates $P<.05$ 


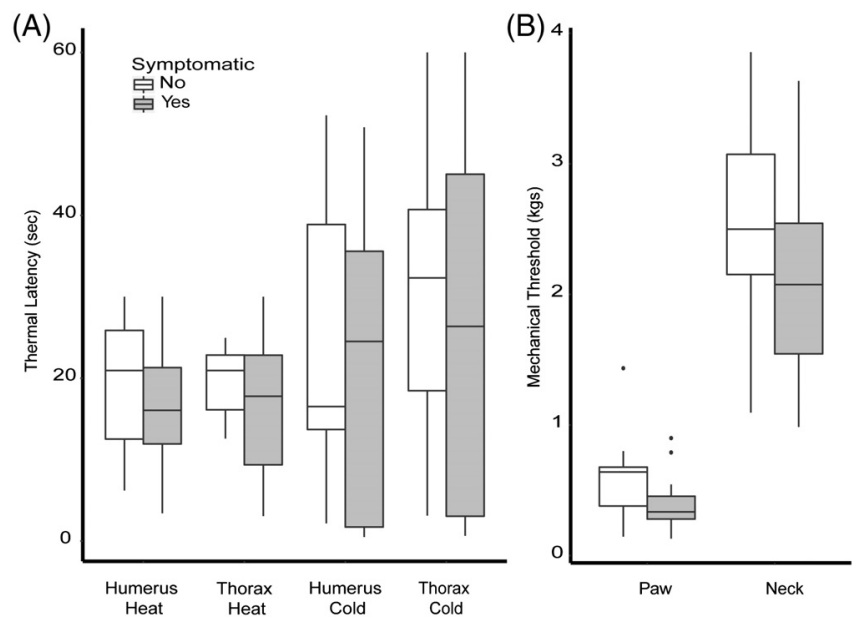

FIGURE 4 Box-plot showing thermal latencies (A) and mechanical thresholds $(B)$ in dogs that were owner-reported symptomatic or asymptomatic. For thermal testing, every location is displayed as an average of left and right sides and triplicate trials per dog. For mechanical testing, left and right sides were averaged and every dog was tested once per location. Refer to Figure 2 legend for a description of the box-plot construct

user. To address this problem, the investigator performing the sensory testing practiced delivering a continuous, increasing force while observing the force recorded on the accompanying software.

Thermal testing also has been used in humans ${ }^{15-17,35}$ and dogs ${ }^{23-26,29}$ to evaluate neuropathic and chronic pain. Temperature and pain stimuli are carried by nonmyelinated $C$ fibers through the spinothalamic pathway. A lightbox model (Canine Thermal Escape Model) has been used that allows latency to heat to be quantified by measuring the time taken for a dog to move its paw away from a light beam directed at the ventral surface of the paw. ${ }^{29}$ Given our desire to test specific dermatome regions, the thermal probe allowed testing of regions pertinent to CKCS with CMSM. Previously established temperatures and maximum duration for testing was determined by 1 of the authors (Duncan Lascelles). Using these methods, we were able to minimize skin irritation, with only 1 dog exhibiting sensitivity to the thermal probe.

Adapting previously described protocols, testing locations for each sensory modality were selected based on clinical signs of CMSM in dogs, neuroanatomy of sensory dermatomes and feasibility in pet dogs. The locations tested were determined by extensive trial and error during development of the protocols and were based on the ability to gather reliable data. Human CM1 patients describe sensory abnormalities affecting the upper extremities and head and neck region. Most commonly, owners of affected CKCS report signs in similar areas including the shoulder, head, and neck regions. Furthermore, CKCS with SM frequently have both cervical and thoracic involvement. Given this information, our testing sites (neck, humerus, and thorax) were located in dermatomes associated with syrinx location. Despite our carefully devised protocol, we found no association between SM and sensory dysfunction. Clearly, SM location along the spinal cord could impact the distribution of hyperesthesia or hypoesthesia, and perhaps sensory testing should be tailored for each individual dog. Because the location of SM can differ among dogs, it is difficult to generate a feasible, reliable sensory testing protocol suitable for all CKCS with SM. Nonetheless, these findings are in agreement with our detailed questionnaire analysis of owner-reported signs in CKCS in the same population of dogs, where it was concluded that the presence of scratching and pain per owner were not associated with $\mathrm{SM}^{8}$

The lack of association between SM and sensory dysfunction may highlight the difficulty in deciphering canine behavior using these protocols. Nonetheless, our feasibility scores demonstrated testing was performed with no difficulty or mild difficulty in $76 \%$ of the dogs tested. Poor feasibility scores were rare $(n=5)$ and were attributed to external distractions, anxiety, or reluctance to be restrained. Another potential pitfall of this work was the number of dogs on medications during the trial. Owners were asked to withdraw medications before testing, but some owners were reluctant to do so because of the severity of their dogs' signs. All of these dogs were reported to be symptomatic while on their medications, but the analgesic drugs they were receiving could have altered the results of the QST. In addition, because of our small sample size ( $n=44$ for mechanical, $n=32$ for thermal) with only 14 CKCS without SM, we may not have had adequate study power to detect differences among groups. We anticipate that these data can be used in future studies to estimate appropriate group sizes. Lastly, we recognize that hyperesthesia could have been caused by coat clipping before thermal testing in some dogs, potentially increasing variability in thermal latencies. Although this lack of association is contrary to previous studies demonstrating a strong relationship between SM and pain, 1,11,36,37 there have been reports of asymptomatic CKCS with SM and CKCS displaying classical clinical signs without SM. ${ }^{8,20,38,39}$ In our study, we had 7 symptomatic dogs without SM and 8 asymptomatic dogs with SM.

Despite the aforementioned pitfalls, we did see a correlation between the presence of pain on neurological examination and decreased mechanical thresholds. Furthermore, neck mechanical testing data were evenly distributed in a population of dogs displaying a wide range of clinical signs. In this manner, the mechanical thresholds determined using our methodology (instrumented forceps) appear to be capturing owner and clinician observations of signs in a more quantitative fashion. These findings may be somewhat limited by individual dog personality type. It is possible that some dogs were more likely to respond to both neck palpation and mechanical thresholds because of distinct personality characteristics rather than sensory abnormalities.

In summary, the purpose of our study was to quantify sensory thresholds in a cohort of CKCS with and without SM. We found no correlation between sensory thresholds (quantified by mechanical thresholds, thermal latencies and mechanical and thermal response rates) and SM. However, the presence or absence of pain on neurological examination did correlate with mechanical thresholds. These tools may be useful for assessing sensory changes in a clinical trial setting. Because of the inconsistencies between the presence and severity of SM and QST data, the relationship between SM and clinical signs deserves further examination.

\section{ACKNOWLEDGMENTS}

The authors thank the owners who allowed their dogs to participate in this study. This study was performed at North Carolina State 
University's College of Veterinary Medicine. This work was funded by a grant from the American Cavalier King Charles Spaniel Club Charitable Trust administered by the AKC Canine Health Foundation. This work was presented at 2016 NCSU CVM Research forum (Raleigh, NC), 2017 Merial NIH Veterinary Scholars Program Symposium (Bethesda, MD), and 2017 Pain in Animals Workshop (Bethesda, MD).

\section{CONFLICT OF INTEREST DECLARATION}

Authors declare no conflict of interest.

\section{OFF-LABEL ANTIMICROBIAL DECLARATION}

Authors declare no off-label use of antimicrobials.

\section{INSTITUTIONAL ANIMAL CARE AND USE COMMITTEE (IACUC) OR OTHER APPROVAL DECLARATION}

IACUC protocol number 15-003-O.

\section{ORCID}

\section{B. Duncan X. Lascelles (D) https://orcid.org/0000-0002-2950-9009} Natasha J. Olby (D) https://orcid.org/0000-0003-1349-3484

\section{REFERENCES}

1. Cerda-Gonzalez S, Olby NJ, McCullough S, et al. Morphology of the Caudal Fossa in Cavalier King Charles Spaniels. Vet Radiol Ultrasound. 2009;50:37-46.

2. Driver CJ, Rusbridge C, Cross HR, McGonnell I, Volk HA. Relationship of brain parenchyma within the Caudal Cranial Fossa and ventricle size to syringomyelia in Cavalier King Charles Spaniels. J Small Anim Pract. 2010;51:382-386.

3. Carruthers H, Rusbridge C, Dubé M-, Holmes M, Jeffery N. Association between cervical and intracranial dimensions and syringomyelia in the Cavalier King Charles Spaniel. J Small Anim Pract 2009; 50: 394-398.

4. Cross HR, Cappello R, Rusbridge C. Comparison of cerebral cranium volumes between Cavalier King Charles Spaniels with Chiari-like malformation, small breed dogs and Labradors. J Small Anim Pract. 2009;50:399-405.

5. Cappello R, Rusbridge C. Report from the Chiari-like malformation and syringomyelia working group round table. Vet Surg. 2007;36:509-512.

6. Rusbridge $\mathrm{C}$. Chiari-like malformation with syringomyelia in the Cavalier King Charles Spaniel: long-term outcome after surgical management. Vet Surg. 2007;36:396-405.

7. Rusbridge C, Knowler SP. Inheritance of occipital bone hypoplasia (Chiari type I malformation) in Cavalier King Charles Spaniels. J Vet Intern Med. 2004;18:673-678.

8. Sparks CR, Cerda-Gonzalez S, Griffith EH, Lascelles BDX, Olby NJ. Questionnaire-based analysis of owner-reported scratching and pain signs in Cavalier King Charles Spaniels screened for Chiari-like malformation and syringomyelia. J Vet Intern Med. 2018;32:331-339.

9. Rusbridge C, Jeffery ND. Pathophysiology and treatment of neuropathic pain associated with syringomyelia. Vet J. 2008;175:164-172.

10. Hu HZ, Rusbridge C, Constantino-Casas F, Jeffery N. Histopathological investigation of syringomyelia in the Cavalier King Charles Spaniel. J Comp Pathol. 2012;146:192-201.

11. Rusbridge $C$, Carruthers $H$, Dubé M-, Holmes M, Jeffery ND. Syringomyelia in Cavalier King Charles Spaniels: the relationship between syrinx dimensions and pain. J Small Anim Pract. 2007; 48:432-436.
12. Ellenbogen RG, Armonda RA, Shaw DWW, Winn HR. Toward a rational treatment of Chiari I malformation and syringomyelia. Neurosurg Focus. 2000;8:1-10.

13. Milhorat TH, Chou MW, Trinidad EM, et al. Chiari I malformation redefined: clinical and radiographic findings for 364 symptomatic patients. Neurosurgery. 1999;44:1005-1017.

14. Fischbein R, Saling J, Marty P, et al. Patient-reported Chiari malformation type I symptoms and diagnostic experiences: a report from the National Conquer Chiari Patient Registry Database. Neurol Sci. 2015; 36:1617-1624.

15. Attal N, Parker F, Tadi e M, Aghakani N, Bouhassira D. Effects of surgery on the sensory deficits of syringomyelia and predictors of outcome: a long term prospective study. J Neurol Neurosurg Psychiatr. 2004;75:1025-1030.

16. Ducreux D, Attal N, Parker F, Bouhassira D. Mechanisms of central neuropathic pain: a combined psychophysical and fMRI study in syringomyelia. Brain. 2006;129:963-976.

17. Thimineur M, Kitaj M, Kravitz E, Kalizewski T, Sood P. Functional abnormalities of the cervical cord and lower medulla and their effect on pain: observations in chronic pain patients with incidental mild Chiari I malformation and moderate to severe cervical cord compression. Clin J Pain. 2002;18:171-179.

18. Geber C, Klein T, Azad S, et al. Test-retest and Interobserver reliability of quantitative sensory testing according to the protocol of the German research network on neuropathic pain (DFNS): a multi-Centre study. Pain. 2011;152:548-556.

19. Plessas IN, Rusbridge C, Driver CJ, et al. Long-term outcome of Cavalier King Charles Spaniel dogs with clinical signs associated with Chiari-like malformation and syringomyelia. Vet Rec. 2012;171:501.

20. Ives EJ, Doyle L, Holmes M, Williams TL, Vanhaesebrouck AE. Association between the findings on magnetic resonance imaging screening for syringomyelia in asymptomatic Cavalier King Charles Spaniels and observation of clinical signs consistent with syringomyelia in later life. Vet J. 2015;203:129-130.

21. Rutherford L, Wessmann A, Rusbridge C, et al. Questionnaire-based behaviour analysis of Cavalier King Charles Spaniels with neuropathic pain due to Chiari-like malformation and syringomyelia. Vet J. 2012; 194:294-298.

22. Braz J, Solorzano $C$, Wang $X$, Basbaum A. Transmitting pain and itch messages: a contemporary view of the spinal cord circuits that generate gate control. Neuron. 2014;82:522-536.

23. Freire M, Knazovicky D, Case B, Thomson A, Lascelles BDX. Comparison of thermal and mechanical quantitative sensory testing in client-owned dogs with chronic naturally occurring pain and normal dogs. Vet J. 2016;210:95-97.

24. Gorney AM, Blau SR, Dohse CS, et al. Mechanical and thermal sensory testing in normal Chondrodystrophoid dogs and dogs with spinal cord injury caused by thoracolumbar intervertebral disc Herniations. J Vet Intern Med. 2016;30:627-635.

25. Knazovicky D, Helgeson ES, Case B, Gruen ME, Maixner W, Lascelles BDX. Widespread somatosensory sensitivity in naturally occurring canine model of osteoarthritis. Pain. 2016;157:1325-1332.

26. Knazovicky D, Helgeson ES, Case B, et al. Replicate effects and testretest reliability of quantitative sensory threshold testing in dogs with and without chronic pain. Vet Anaesth Analg. 2017;44:615-624.

27. KuKanich B, Lascelles BD, Papich MG. Assessment of a Von Frey device for evaluation of the antinociceptive effects of morphine and its application in pharmacodynamic modeling of morphine in dogs. Am J Vet Res. 2005;66:1616-1622

28. KuKanich B, Lascelles BD, Papich MG. Use of a Von Frey device for evaluation of pharmacokinetics and pharmacodynamics of morphine after intravenous administration as an infusion or multiple doses in dogs. Am J Vet Res. 2005;66:1968-1974.

29. Williams MD, Kirkpatrick AE, Griffith E, Benito J, Hash J, Lascelles BDX. Feasibility and repeatability of thermal quantitative sensory testing in normal dogs and dogs with hind limb osteoarthritisassociated pain. Vet J. 2014;199:63-67.

30. Trumpatori BJ, Carter JE, Hash J, et al. Evaluation of a Midhumeral block of the radial, ulnar, musculocutaneous and median (RUMM block) nerves for analgesia of the distal aspect of the thoracic limb in dogs. Vet Surg. 2010;39:785-796. 
31. Sanchis-Mora S, Chang YM, Abeyesinghe S, Fisher A, Volk HA, Pelligand L. Development and initial validation of a sensory threshold examination protocol (STEP) for phenotyping canine pain syndromes. Vet Anaesth Analg. 2017;44:600-614.

32. Moore SA, Hettlich BF, Waln A. The use of an electronic Von Frey device for evaluation of sensory threshold in neurologically normal dogs and those with acute spinal cord injury. Vet J. 2013;197:216-219.

33. Briley JD, Williams MD, Freire M, Griffith EH, Lascelles BDX. Feasibility and repeatability of cold and mechanical quantitative sensory testing in normal dogs. Vet J. 2014;199:245-250.

34. Rolke R, Baron R, Maier C, et al. Quantitative sensory testing in the German research network on neuropathic pain (DFNS): standardized protocol and reference values. Pain. 2006;123:231-243.

35. Verdugo R, Ochoa JL. Quantitative somatosensory thermotest. A key method for functional evaluation of small calibre afferent channels. Brain. 1992;115 (Pt 3:893-913.

36. Hu HZ, Rusbridge C, Constantino-Casas F, Jeffery N. Distribution of substance $\mathrm{P}$ and calcitonin gene-related peptide in the spinal cord of Cavalier King Charles Spaniels affected by symptomatic syringomyelia. Res Vet Sci. 2012;93:318-320.

37. Schmidt MJ, Roth J, Ondreka N, Kramer M, Rummel C. A potential role for substance $P$ and Interleukin-6 in the cerebrospinal fluid of Cavalier King Charles Spaniels with neuropathic pain. J Vet Intern Med. 2013;27:530-535.
38. Thøfner MS, Stougaard CL, Westrup U, et al. Prevalence and heritability of symptomatic syringomyelia in cavalier king Charles spaniels and long-term outcome in symptomatic and asymptomatic littermates. J Vet Intern Med. 2015;29:243-250.

39. Parker JE, Knowler SP, Rusbridge C, Noorman E, Jeffery ND. Prevalence of asymptomatic syringomyelia in Cavalier King Charles Spaniels. Vet Rec. 2011;168:667-667.

\section{SUPPORTING INFORMATION}

Additional supporting information may be found online in the Supporting Information section at the end of the article.

How to cite this article: Sparks CR, Gorney A, Williams K, et al. Investigation of sensory thresholds in Cavalier King Charles Spaniels with and without Chiari-like malformations and syringomyelia. J Vet Intern Med. 2018;32:2021-2028. https://doi.org/10.1111/jvim.15297 